\title{
ANALYSIS OF THE FINITE ELEMENT APPROXIMATION OF MICROSTRUCTURE IN MICROMAGNETICS
}

\author{
By \\ Mitchell Luskin \\ and \\ Ling Ma
}

IMA Preprint Series \# 740

December 1990 


\title{
ANALYSIS OF THE FINITE ELEMENT APPROXIMATION OF MICROSTRUCTURE IN MICROMAGNETICS*
}

\author{
MITCHELL LUSKIN† AND LING MA
}

\begin{abstract}
The solution to nonconvex variational problems is often characterized by microstructure. The variational problem for the magnetization field in micromagnetics has a nonconvex constraint and energy minimizing sequences of magnetization fields can have oscillations whose scale converges to zero but whose amplitude remains finite. We show that the finite element approximation of the magnetization field for this variational problem does not converge pointwise as the mesh is refined, but that nonlinear functions of the approximate magnetization fields converge weakly (or equivalently, all local spatial averages of nonlinear functions of the approximate magnetization field converge as the mesh is refined, which implies the convergence as the mesh is refined of the probability distribution of the approximate magnetization fields in local spatial domains). We give a norm to measure the convergence of this microstructure, and we prove a rate of convergence in this norm.
\end{abstract}

Key words. finite element method, microstructure, micromagnetics, magnetization field

AMS(MOS) subject classifications. $65 \mathrm{~N} 15,65 \mathrm{~N} 30,35 \mathrm{~J} 20,35 \mathrm{~J} 70,73 \mathrm{C} 60$

1. Introduction. There has been great progress during the past several years in the analysis and computation of nonconvex variational problems which are important mathematical models in many areas of science and technology [4-5, 9-18, 20-29]. The solutions to these variational problems often are characterized by a microstructure [4-5, 11, 23-28]. In $[12,15-16]$, we introduced new concepts to analyze the finite element approximation of the microstructure found in martensitic materials. In this paper, we show how these concepts can be utilized to analyze the finite element approximation of the magnetization field for some ferromagnetic materials which exhibit microstructure in the form of fine-scale magnetic domains.

The magnetization field of many ferromagnetic materials has a fine scale structure or a microstructure. This microstructure is modeled by the theory of micromagnetics [6-8]. The theory of micromagnetics provides a free energy density for the magnetization field, and magnetization fields with microstructure minimize the bulk energy [25-26].

The micromagnetics variational problem is a nonconvex variational problem, and it has been recently shown that these variational problems can fail to attain a minimum value for any classical function [25-26]. Minimizing sequences of magnetization fields can have

*This work is part of the Transitions and Defects in Ordered Materials Project and was supported in part by the National Science Foundation and the Air Force Office of Scientific Research through grants DMS 835-1080 and DMS 871-8881, the Army Research Office through grants DAAL03-88-K-0110 and DAAL03-89-G-0081, the Cray Research Foundation, and by a grant from the Minnesota Supercomputer Institute.

†School of Mathematics, University of Minnesota, Minneapolis, Minnesota 55455.

$\ddagger$ School of Mathematics, University of Minnesota, Minneapolis, Minnesota 55455. 
oscillations whose scale converges to zero but whose amplitude remains finite. Numerical approximations of such fields will clearly not converge in any $L^{p}$ space as the mesh is refined, even locally. However, we demonstrate in this paper that nonlinear functions of the approximate magnetization fields converge weakly, i.e., all local spatial averages of nonlinear functions of the approximate magnetization field converge as the mesh is refined, which implies the convergence as the mesh is refined of the probability distribution of the approximate magnetization fields in local spatial domains [2]. To give error estimates for the convergence of this microstructure, we utilize the norm that we introduced in [16] to analyze the numerical approximation of martensitic microstructure. This theory validates the computational results for the microstructure in micromagnetics reported in [29].

2. The Theory of Micromagnetics. We denote the magnetization field by $m$ : $\Omega \rightarrow \mathbb{R}^{3}$ where $\Omega \subseteq \mathbb{R}^{3}$ represents the spatial domain of the ferromagnetic material with boundary $\partial \Omega$. We assume for simplicity that the boundary $\partial \Omega$ is the union of triangular faces $\Gamma_{l}$ which are defined as the convex hull of noncollinear points $\left\{a_{l, 1}, a_{l, 2}, a_{l, 3}\right\} \subset \mathbb{R}^{3}$ or

$$
\Gamma_{l}=\left\{x=\sum_{i=1}^{3} \beta_{i} \alpha_{l, i}: \beta_{i} \geq 0, \sum_{i=1}^{3} \beta_{i}=1\right\} .
$$

Further, we have that

$$
\partial \Omega=\bigcup_{l=1}^{L} \Gamma_{l} \quad \text { and } \quad \text { int } \Gamma_{l} \cap \operatorname{int} \Gamma_{j}=\emptyset \text { if } l \neq j
$$

where int $\Gamma_{l}$ is the interior of $\Gamma_{l}$.

We assume that the material is magnetically saturated, so that

$$
|m(x)|=f(\theta) \quad x \in \Omega
$$

where $\theta$ is the temperature and || denotes the Euclidean norm. Since we shall not be concerned here with temperature variations, we can assume without loss of generality that

$$
|m(x)|=1 \quad x \in \Omega .
$$

The free energy for the magnetization field is the sum of the anisotropic energy of the crystal and the magnetostatic energy. The anisotropic energy density $\phi(m): \mathbb{S}^{2} \rightarrow \mathbb{R}$ where

$$
\mathbb{S}^{2}=\left\{m \in \mathbb{R}^{3}:|m|=1\right\}
$$

has the material symmetry property

$$
\phi(R m)=\phi(m) \quad R \in \mathcal{G}
$$


where $\mathcal{G}$ is the symmetry group of the crystal. In this paper, we shall be concerned only with the uniaxial case (for example, cobalt) where $\phi(m)$ is even and where there is a unit vector $\tilde{m}$ such that

$$
0=\phi( \pm \tilde{m})<\phi(m) \quad \text { for all } m \neq \pm \tilde{m} .
$$

More precisely, we shall assume that there exists a positive constant $c_{0}$ such that

$$
\begin{aligned}
& \phi(m) \geq c_{0} \inf \left(|m-\tilde{m}|^{2},|m-(-\tilde{m})|^{2}\right) \\
& \phi(\tilde{m})=0 .
\end{aligned}
$$

We will also assume without loss of generality that $\tilde{m}=e_{3}=(0,0,1)$.

The magnetostatic energy density is given by $|H|^{2} / 2$ where $H: \mathbb{R}^{3} \rightarrow \mathbb{R}^{3}$ is the magnetic field. Maxwell's equations in dimensionless units are given by

$$
\operatorname{div} B=0 \quad \text { and } \quad \operatorname{curl} H=0
$$

where $B=H+m: \mathbb{R}^{3} \rightarrow \mathbb{R}^{3}$ is the magnetic induction. So, the magnetic potential $u: \mathbb{R}^{3} \rightarrow \mathbb{R}$ where $H=-\nabla u$ must satisfy the partial differential equation

$$
\operatorname{div}\left(-\nabla u+m \chi_{\Omega}\right)=0 \quad \text { in } \mathbb{R}^{3}
$$

where $\chi_{\Omega}(x)$ is the characteristic function of $\Omega$ defined by

$$
\chi_{\Omega}(x)=\left\{\begin{array}{l}
1 \text { if } x \in \Omega \\
0 \text { if } x \notin \Omega .
\end{array}\right.
$$

Thus,

$$
m(x) \chi_{\Omega}(x)=\left\{\begin{array}{r}
m(x) \text { if } x \in \Omega \\
0 \text { if } x \notin \Omega .
\end{array}\right.
$$

It is easy to verify by using the Fourier transform that since $m(x) \chi_{\Omega}(x) \in L^{2}\left(\mathbb{R}^{3} ; \mathbb{R}^{3}\right) \cap$ $L^{1}\left(\mathbb{R}^{3} ; \mathbb{R}^{3}\right),(2.3)$ has a unique solution $u(x) \in H^{1}\left(\mathbb{R}^{3}\right)[2,19]$.

We sum the contributions of the anisotropic energy and the magnetostatic energy to obtain the bulk energy for the magnetization field given by

$$
E(m)=\int_{\Omega} \phi(m(x)) d x+\frac{1}{2} \int_{\mathbb{R}^{3}}|\nabla u|^{2} d x \quad \text { for } m \in \mathcal{A}
$$

where

$$
\operatorname{div}\left(-\nabla u+m \chi_{\Omega}\right)=0 \quad \text { in } \mathbb{R}^{3}
$$

and where

$$
\mathcal{A}=\left\{m \in L^{\infty}\left(\Omega ; \mathbb{R}^{3}\right):|m(x)|=1 \text { a.e. in } \Omega\right\} .
$$


It is shown in [25-26] that

$$
\inf _{m \in \mathcal{A}} E(m)=0
$$

However, it is also shown in [25-26] that there does not exist $m \in \mathcal{A}$ such that $E(m)=0$. The direct method of the calculus of variations does not give a magnetization field at which the bulk energy functional (2.4) attains the minimum value even though the bulk energy functional is weakly continuous (since the bulk energy functional is convex). The weak limit of a minimizing sequence of magnetization fields is the zero field $m \equiv 0$ which is not in the set of admissible fields $\mathcal{A}$ (The set of admissible functions (2.5) is not weakly closed.)

Sometimes an "exchange energy" is also included in the bulk energy to account for the tendency of the magnetic moments to align. However, the exchange energy has a negligible effect on the macroscopic magnetic properties of the material, and we therefore neglect this term.

3. The Numerical Approximation and the Statement of the Results. In this section, we define our finite element approximation of the magnetization field. For $h>0$, we define

$$
I_{i j k}=(i h,(i+1) h) \times(j h,(j+1) h) \times(k h,(k+1) h), \quad i, j, k \in \mathbb{Z},
$$

and we define the decomposition of $\Omega$ into disjoint sets

$$
\Omega_{i j k}=I_{i j k} \cap \Omega \quad i, j, k \in \mathbb{Z} \text {. }
$$

We note that $\Omega_{i j k} \neq \emptyset$ for a finite set of $i, j, k \in \mathbb{Z}$.

Our finite element method is to minimize the bulk energy on the piecewise constant space

$$
\mathcal{A}_{h}=\left\{m \in \mathcal{A}: m(x) \text { is constant on } \Omega_{i j k} \text { for } i, j, k \in \mathbb{Z}\right\} \text {. }
$$

The finite element approximation is given by $m_{h} \in \mathcal{A}_{h}$ such that

$$
E\left(m_{h}\right)=\inf _{m \in \mathcal{A}_{h}} E(m)
$$

We note that (3.1) attains a minimum value since the energy is non-negative and since the dimension of $\mathcal{A}_{h}$ is finite. The minimum energy for the finite element approximation (3.1) can be attained by more than one approximate magnetization field, though.

Our first result gives an estimate of the energy of finite element approximations given by (3.1). 
THEOREM 1. There exists a constant $c_{1}$, independent of $h$, such that if the magnetization field $m_{h} \in \mathcal{A}_{h}$ is a solution to the finite element variational problem (3.1), then

$$
E\left(m_{h}\right)=\inf _{m \in \mathcal{A}_{h}} E(m) \leq c_{1} h
$$

Our second main theorem states that the approximate magnetization fields $m_{h}(x)$ are locally in the state $e_{3}$ with probability $1 / 2$ and locally in the state $-e_{3}$ with probability $1 / 2$. In the jargon of the calculus of variations, the measure

$$
\nu_{x} \equiv \frac{1}{2} \delta_{e_{3}}+\frac{1}{2} \delta_{-e_{3}} \quad \text { for } x \in \Omega
$$

where $\delta_{\hat{m}}$ is the Dirac delta function with unit mass concentrated at $\hat{m}$ for $\hat{m}=e_{3},-e_{3}$ is the unique Young measure associated with minimizing $(3.1)[3,5,32]$.

We define the Sobolev Space $[1,2,19] \mathcal{U}$ to be the closure of the space $C_{c}^{\infty}(\Omega)$, the space of $C^{\infty}$ functions on $\Omega$ with compact support in $\Omega$, with respect to the norm

$$
\|\zeta\|_{\mathcal{U}}^{2}=\|\zeta\|_{L^{2}(\Omega)}^{2}+\left\|\zeta_{x_{1}}\right\|_{L^{2}(\Omega)}^{2}+\left\|\zeta_{x_{2}}\right\|_{L^{2}(\Omega)}^{2}
$$

and we define $\operatorname{Lip}\left(\mathbb{S}^{2}\right)$ to be space of Lipschitz continuous functions $\psi: \mathbb{S}^{2} \rightarrow \mathbb{R}$ with semi-norm

$$
\|\psi\|_{L i p\left(\mathbb{S}^{2}\right)}=\sup _{\substack{p, q \in \mathbb{S}^{2} \\ p \neq q}} \frac{|\psi(p)-\psi(q)|}{|p-q|}
$$

Next, we define the vector space $\mathcal{V}$ to be the space of functions $F(x, m): \Omega \times \mathbb{S}^{2} \rightarrow \mathbb{R}$ such that

$$
F(x, m) \in L^{2}\left(\Omega ; \operatorname{Lip}\left(\mathbb{S}^{2}\right)\right)
$$

and

$$
F\left(x, e_{3}\right)-F\left(x,-e_{3}\right) \in \mathcal{U}
$$

with semi-norm

$$
\|F\|_{\mathcal{V}}=\left[\int_{\Omega}\|F(x, \cdot)\|_{L i p\left(\mathbb{S}^{2}\right)}^{2} d x\right]^{1 / 2}+\left\|F\left(x, e_{3}\right)-F\left(x,-e_{3}\right)\right\|_{\mathcal{U}} .
$$

We will prove the following theorem which gives the weak limit of nonlinear functions of $m_{h}$.

THEOREM 2. We have that there exists a constant $c_{2}$, independent of $h$, such that if the magnetization field $m_{h} \in \mathcal{A}_{h}$ is a solution to the finite element variational problem (3.1), then

$$
\begin{gathered}
\left|\int_{\Omega} F\left(x, m_{h}\right) d x-\frac{1}{2} \int_{\Omega}\left[F\left(x, e_{3}\right)+F\left(x,-e_{3}\right)\right] d x\right| \\
\leq c_{2} h^{1 / 2}\|F\|_{\mathcal{V}} \quad \text { for all } F \in \mathcal{V} .
\end{gathered}
$$


We note that the mechanical properties of magnetic materials depend nonlinearly on the magnetization $m$. Theorem 2 shows that the material property described by the microscopic density $F(x, m)$ has the macroscopic density (weak limit)

$$
\frac{1}{2} F\left(x, e_{3}\right)+\frac{1}{2} F\left(x,-e_{3}\right)
$$

for the minimizing microstructure for the energy (3.1).

To estimate the rate of convergence of $m_{h}(x)$ we define the dual of $\mathcal{V}, \mathcal{V}^{*}$, to be the set of linear functionals $L: \mathcal{V} \rightarrow \mathbb{R}$ for which there exists a constant $c$ such that

$$
|\langle L, F\rangle| \leq c\|F\|_{\mathcal{V}} \quad \text { for all } F \in \mathcal{V}
$$

with operator norm [30] defined for $L \in \mathcal{V}^{*}$ by

$$
\|L\|_{\mathcal{V}^{*}}=\sup _{\substack{F \in \mathcal{V} \\\|F\|_{\mathcal{V}=1}}}|\langle L, F\rangle|
$$

We can identify with $m_{h}$ the functional $L_{m_{h}} \in \mathcal{V}^{*}$ defined by

$$
\left\langle L_{m_{h}}, F\right\rangle \equiv \int_{\Omega} F\left(x, m_{h}(x)\right) d x
$$

and we identify with $\nu \equiv \frac{1}{2} \delta_{e_{3}}+\frac{1}{2} \delta_{-e_{3}}$ the functional $L_{\nu} \in \mathcal{V}^{*}$ defined by

$$
\left\langle L_{\nu}, F\right\rangle \equiv \frac{1}{2} \int_{\Omega}\left[F\left(x, e_{3}\right)+F\left(x,-e_{3}\right)\right] d x
$$

We then have the following theorem which is a direct consequence of (3.3).

THEOREM 3. We have the error estimate

$$
\left\|L_{m_{h}}-L_{\nu}\right\|_{\mathcal{V}^{*}} \leq c_{2} h^{1 / 2}
$$

4. Proofs of the Main Theorems. We first give the proof of Theorem 1 .

Proof of Theorem 1. We define $p_{h}(x) \in \mathcal{A}_{h}$ by

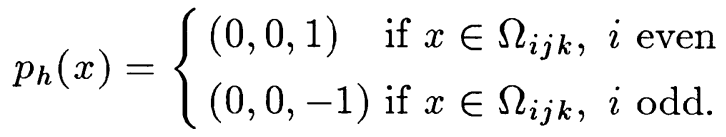

Then $\phi\left(p_{h}(x)\right)=0$ for $x \in \Omega$ and

$$
E\left(p_{h}\right)=\frac{1}{2} \int_{\mathbb{R}^{3}}|\nabla u|^{2} d x .
$$


Now by $(2.3)$

$$
\frac{1}{2} \int_{\mathbb{R}^{3}}|\nabla u|^{2} d x=\frac{1}{2} \int_{\Omega} \nabla u \cdot p_{h} d x .
$$

Further, $u \in H^{1}\left(\mathbb{R}^{3}\right)$ satisfies

$$
u=\operatorname{div} w
$$

where $w=\left(w_{1}, w_{2}, w_{3}\right) \in C^{1}\left(\mathbb{R}^{3} ; \mathbb{R}^{3}\right)$ with $\nabla w \in H^{1}\left(\mathbb{R}^{3 \times 3} ; \mathbb{R}^{3 \times 3}\right)$ is uniquely defined by [19]

$$
\begin{aligned}
& \Delta w=p_{h} \chi_{\Omega}, \\
& w(x) \rightarrow 0 \text { as }|x| \rightarrow \infty .
\end{aligned}
$$

Note that $w_{1}(x)=w_{2}(x)=0$ for all $x \in \mathbb{R}^{3}$. Hence,

$$
\nabla u \cdot p_{h}=w_{3,33} P_{h}
$$

where $P_{h}: \bar{\Omega} \rightarrow \mathbb{R}$ satisfies

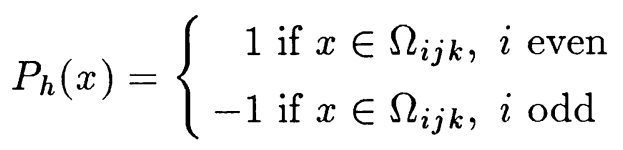

and is extended naturally to $\partial \Omega$. Thus, by the divergence theorem

$$
\frac{1}{2} \int_{\Omega} \nabla u \cdot p_{h} d x=\frac{1}{2} \int_{\Omega} w_{3,33} P_{h} d x=\frac{1}{2} \int_{\partial \Omega} w_{3,3} P_{h} n_{3} d S
$$

where $n=\left(n_{1}, n_{2}, n_{3}\right)=n(S)$ is the unit exterior normal to $\partial \Omega$ at $S \in \partial \Omega$ and $d S$ is the surface area measure on $\partial \Omega$.

By (4.1) and (4.2),

$$
w_{3}(x)=-\frac{1}{4 \pi} \int_{\Omega} \frac{P_{h}(y)}{|x-y|} d y
$$

So,

$$
\begin{aligned}
w_{3,3}(x) & =-\frac{1}{4 \pi} \int_{\Omega} P_{h}(y) \frac{\partial}{\partial x_{3}} \frac{1}{|x-y|} d y \\
& =\frac{1}{4 \pi} \int_{\partial \Omega} \frac{P_{h}(S) n_{3}}{|x-S|} d S .
\end{aligned}
$$

Since $1 /|x-S| \in L^{1}(\partial \Omega)$ for $x \in \partial \Omega$, it follows from (4.3) that there exists a constant $c_{3}$ such that

$$
\left\|w_{3,3}\right\|_{L^{\infty}(\partial \Omega)} \leq c_{3}\left\|P_{h}\right\|_{L^{\infty}(\partial \Omega)} \leq c_{3} .
$$


Let

$$
w_{3,3}^{\Gamma_{l}}(x)=\frac{1}{4 \pi} \int_{\Gamma_{l}} \frac{P_{h}(S) n_{3}}{|x-S|} d S \quad \text { for } x \in \mathbf{R}^{3} .
$$

It follows from the theory of singular integrals [31] that for $1<p<\infty$ there exists a constant $c_{4}=c_{4}(p)$ such that

$$
\left\|\nabla_{S} w_{3,3}^{\Gamma_{l}}\right\|_{L^{p}\left(\Gamma_{l}\right)} \leq c_{4}\left\|P_{h}\right\|_{L^{p}\left(\Gamma_{l}\right)}
$$

where $\nabla_{S}$ denotes the projection of the gradient onto the tangent plane to $\Gamma_{l}$. Thus, for $q=p /(p-1)$ we obtain by (4.5) and Hölder's inequality that

$$
\begin{aligned}
\left\|\nabla_{S} w_{3,3}^{\Gamma_{l}}\right\|_{L^{1}\left(\Gamma_{l}\right)} & \leq \operatorname{area}\left(\Gamma_{l}\right)^{1 / q}\left\|\nabla_{S} w_{3,3}^{\Gamma_{l}}\right\|_{L^{p}\left(\Gamma_{l}\right)} \\
& \leq c_{4} \operatorname{area}\left(\Gamma_{l}\right)^{1 / q}\left\|P_{h}\right\|_{L^{p}\left(\Gamma_{l}\right)} \\
& \leq c_{4} \operatorname{area}\left(\Gamma_{l}\right)\left\|P_{h}\right\|_{L^{\infty}\left(\Gamma_{l}\right)} .
\end{aligned}
$$

Now

$$
\nabla w_{3,3}^{\Gamma_{l}}(x)=-\frac{1}{4 \pi} \int_{\Gamma_{l}} \frac{P_{h}(S) n_{3}}{|x-S|^{3}}(x-S) d S \quad \text { for } x \in \mathbb{R}^{3}-\Gamma_{l},
$$

so there exists a constant $c_{5}$ such that for $x \in \mathbb{R}^{3}-\Gamma_{l}$,

$$
\begin{aligned}
\left|\nabla w_{3,3}^{\Gamma_{l}}(x)\right| & \leq \frac{1}{4 \pi}\left[\int_{\Gamma_{l}} \frac{1}{|x-S|^{2}} d S\right]\left\|P_{h}\right\|_{L^{\infty}\left(\Gamma_{l}\right)} \\
& \leq c_{5}\left[\log \left(\left|\operatorname{dist}\left(x, \Gamma_{l}\right)\right|\right)+1\right]\left\|P_{h}\right\|_{L^{\infty}\left(\Gamma_{l}\right)} \\
& \leq c_{5}\left[\log \left(\left|\operatorname{dist}\left(x, \Gamma_{l}\right)\right|\right)+1\right]
\end{aligned}
$$

where

$$
\operatorname{dist}\left(x, \Gamma_{l}\right)=\inf _{S \in \Gamma_{l}}|x-S|
$$

Thus, we can obtain from integrating (4.7) that

$$
\left\|\nabla_{S} w_{3,3}^{\Gamma_{l}}\right\|_{L^{1}\left(\Gamma_{l^{\prime}}\right)} \leq c_{6}\left\|P_{h}\right\|_{L^{\infty}\left(\Gamma_{l}\right)} \leq c_{6} \quad \text { for all } l^{\prime} \neq l
$$

Hence, it follows from (4.6), (4.8), and the triangle inequality that there exists a constant $c_{7}$ such that

$$
\left\|\nabla_{S} w_{3,3}\right\|_{L^{1}(\partial \Omega)} \leq c_{7}\left\|P_{h}\right\|_{L^{\infty}(\partial \Omega)} \leq c_{7}
$$

We can order the sets $\Gamma_{l}$ so that

$$
\left\{\begin{aligned}
\left|n_{3}\right| & >0 \text { for } S \in \Gamma_{l}, l=1, \ldots, L_{1} \\
n_{3} & =0 \text { for } S \in \Gamma_{l}, l=L_{1}+1, \ldots, L
\end{aligned}\right.
$$


Then

$$
\partial \Omega=\Gamma \cup \Gamma^{\perp} \quad \text { and } \quad \operatorname{int} \Gamma \cap \operatorname{int} \Gamma^{\perp}=\emptyset,
$$

where

$$
\Gamma=\left\{S \in \partial \Omega:\left|n_{3}\right|>0\right\}=\bigcup_{l=1}^{L_{1}} \Gamma_{l}
$$

and

$$
\Gamma^{\perp}=\left\{S \in \partial \Omega:\left|n_{3}\right|=0\right\}=\bigcup_{l=L_{1}+1}^{L} \Gamma_{l} .
$$

Thus,

$$
\begin{aligned}
\frac{1}{2} \int_{\partial \Omega} w_{3,3} P_{h} n_{3} d S & =\frac{1}{2} \int_{\Gamma} w_{3,3} P_{h} n_{3} d S+\frac{1}{2} \int_{\Gamma^{\perp}} w_{3,3} P_{h} n_{3} d S \\
& =\frac{1}{2} \int_{\Gamma} w_{3,3} P_{h} n_{3} d S
\end{aligned}
$$

For $l=1, \ldots, L_{1}$ there exists affine functions $y_{l}: \tilde{\Gamma}_{l} \subseteq \mathbb{R}^{2} \rightarrow \mathbb{R}$ and isomorphisms $\phi_{l}(x): \tilde{\Gamma}_{l} \subseteq \mathrm{R}^{2} \rightarrow \Gamma_{l}$ such that

$$
\tilde{\Gamma}_{l} \equiv\left\{\left(x_{1}, x_{2}\right) \in \mathbb{R}^{2}:\left(x_{1}, x_{2}, x_{3}\right) \in \Gamma_{l}\right\}
$$

and

$$
\phi_{l}\left(\xi_{1}, \xi_{2}\right)=\left(\xi_{1}, \xi_{2}, y_{l}\left(\xi_{1}, \xi_{2}\right)\right) .
$$

Now

$$
\int_{\Gamma} w_{3,3} P_{h} n_{3} d S=\sum_{l=1}^{L_{1}} \int_{\Gamma_{l}} w_{3,3} P_{h} n_{3} d S
$$

and

$$
\int_{\Gamma_{l}} w_{3,3} P_{h}\left|n_{3}\right| d S=\int_{\tilde{\Gamma}_{l}}\left(w_{3,3} \circ \phi_{l}\right)\left(P_{h} \circ \phi_{l}\right) d \xi .
$$

Next, for $i$ an even integer and $j \in \mathbb{Z}$ set

$$
\hat{I}_{i, j} \equiv(i h,(i+2) h) \times(j h,(j+1) h)
$$

and define

$$
\hat{\Lambda}_{h, l} \equiv \bigcup_{\hat{I}_{i, j} \subset \tilde{\Gamma}_{l}} \hat{I}_{i, j}
$$


and

$$
\tilde{\Lambda}_{h, l} \equiv \tilde{\Gamma}_{l}-\hat{\Lambda}_{h, l}
$$

Now for $\hat{I}_{i, j} \subset \tilde{\Gamma}_{l}$, we have that

$$
\begin{aligned}
\mid \int_{\hat{I}_{i, j}}\left(w_{3,3} \circ \phi_{l}\right) & \left(P_{h} \circ \phi_{l}\right) d \xi \mid \\
& =\left|\int_{j h}^{(j+1) h} \int_{i h}^{(i+1) h}\left[w_{3,3} \circ \phi_{l}\left(\xi_{1}+h, \xi_{2}\right)-w_{3,3} \circ \phi_{l}\left(\xi_{1}, \xi_{2}\right)\right] d \xi_{2} d \xi_{1}\right| \\
& \leq h \int_{j h}^{(j+1) h} \int_{i h}^{(i+2) h}\left|\frac{\partial w_{3,3} \circ \phi_{l}}{\partial \xi_{1}}\left(\xi_{1}, \xi_{2}\right)\right| d \xi_{2} d \xi_{1} .
\end{aligned}
$$

Thus,

$$
\begin{aligned}
& \left|\int_{\tilde{\Gamma}_{l}}\left(w_{3,3} \circ \phi_{l}\right)\left(P_{h} \circ \phi_{l}\right) d \xi\right| \\
& \quad \leq\left|\int_{\hat{\Lambda}_{h, l}}\left(w_{3,3} \circ \phi_{l}\right)\left(P_{h} \circ \phi_{l}\right) d \xi\right|+\left|\int_{\tilde{\Lambda}_{h, l}}\left(w_{3,3} \circ \phi_{l}\right)\left(P_{h} \circ \phi_{l}\right) d \xi\right| \\
& \quad \leq h \int_{\hat{\Lambda}_{h, l}}\left|\frac{\partial w_{3,3} \circ \phi_{l}}{\partial \xi_{1}}\right| d \xi+\int_{\tilde{\Lambda}_{h, l}}\left|\left(w_{3,3} \circ \phi_{l}\right)\left(P_{h} \circ \phi_{l}\right)\right| d \xi .
\end{aligned}
$$

Also, since $\left(1,0, \frac{\partial y_{l}}{\partial \xi_{1}}\right)$ is in the tangent plane to $\Gamma_{l}$, we have that

$$
\left(\nabla w_{3,3} \circ \phi_{l}\right) \cdot\left(1,0, \frac{\partial y_{l}}{\partial \xi_{1}}\right)=\left(\nabla_{S} w_{3,3} \circ \phi_{l}\right) \cdot\left(1,0, \frac{\partial y_{l}}{\partial \xi_{1}}\right) .
$$

Hence, by the chain rule

$$
\begin{aligned}
\left|\frac{\partial w_{3,3} \circ \phi_{l}}{\partial \xi_{1}}\right| & =\left|\left(\nabla w_{3,3} \circ \phi_{l}\right) \cdot\left(1,0, \frac{\partial y_{l}}{\partial \xi_{1}}\right)\right| \\
& =\left|\left(\nabla_{S} w_{3,3} \circ \phi_{l}\right) \cdot\left(1,0, \frac{\partial y_{l}}{\partial \xi_{1}}\right)\right| \\
& \leq\left|\nabla_{S} w_{3,3} \circ \phi_{l}\right| \sqrt{1+\left(\frac{\partial y_{l}}{\partial \xi_{1}}\right)^{2}}
\end{aligned}
$$

so

$$
\begin{aligned}
\int_{\hat{\Lambda}_{h, l}} & \left|\frac{\partial w_{3,3} \circ \phi_{l}}{\partial \xi_{1}}\right| d \xi \leq \int_{\hat{\Lambda}_{h, l}}\left|\nabla_{S} w_{3,3} \circ \phi_{l}\right| \sqrt{1+\left(\frac{\partial y_{l}}{\partial \xi_{1}}\right)^{2}} d \xi \\
& \leq \int_{\hat{\Lambda}_{h, l}}\left|\nabla_{S} w_{3,3} \circ \phi_{l}\right| \sqrt{1+\left(\frac{\partial y_{l}}{\partial \xi_{1}}\right)^{2}+\left(\frac{\partial y_{l}}{\partial \xi_{2}}\right)^{2}} d \xi \\
& =\int_{\phi_{l}\left(\hat{\Lambda}_{h, l}\right)}\left|\nabla_{S} w_{3,3}\right| d S \\
& \leq \int_{\Gamma_{l}}\left|\nabla_{S} w_{3,3}\right| d S .
\end{aligned}
$$


Now

$$
\operatorname{area}\left(\tilde{\Lambda}_{h, l}\right) \leq \sqrt{5} h \text { perimeter } \tilde{\Gamma}_{l} \leq \sqrt{5} h \text { perimeter } \Gamma_{l} .
$$

Thus, by (4.11), (4.12), (4.13), and (4.15)

$$
\begin{aligned}
\left|\int_{\Gamma} w_{3,3} P_{h} n_{3} d S\right| & \leq h \sum_{l=1}^{L_{1}} \int_{\Gamma_{l}}\left|\nabla_{S} w_{3,3}\right| d S+\sum_{l=1}^{L_{1}} \int_{\tilde{\Lambda}_{h, l}}\left|\left(w_{3,3} \circ \phi_{l}\right)\left(P_{h} \circ \phi_{l}\right)\right| d \xi \\
& \leq h\left\|\nabla_{S} w_{3,3}\right\|_{L^{1}(\partial \Omega)}+\sum_{l=1}^{L_{1}} \sqrt{5} h \text { perimeter } \Gamma_{l}\left\|w_{3,3}\right\|_{L^{\infty}(\partial \Omega)} .
\end{aligned}
$$

It follows from the above equation and (4.4) and (4.9) that

$$
\left|\int_{\Gamma} w_{3,3} P_{h} n_{3} d S\right| \leq\left(c_{7}+\sqrt{5} c_{3} \sum_{l=1}^{L_{1}} \text { perimeter } \Gamma_{l}\right) h
$$

The theorem follows from (4.16) and (4.10).

Define the operator $\Pi: \mathcal{A} \rightarrow \mathcal{A}$ by

$$
\Pi m(x)=\left(0,0, \operatorname{sign} m_{3}(x)\right) \quad \text { for } x \in \Omega,
$$

where $m=\left(m_{1}, m_{2}, m_{3}\right)$. Note that

$$
|m-\Pi m|=\inf \left(\left|m-e_{3}\right|,\left|m-\left(-e_{3}\right)\right|\right) .
$$

The following two lemmas will be used in the proof of Theorem 2 .

LEMma 1. There exists a constant $c_{8}$, independent of $h$, such that

$$
\int_{\Omega}\left|m_{h}-\Pi m_{h}\right|^{2} d x \leq c_{8} h
$$

Proof of Lemma 1. It follows from (2.2), (2.4), and Theorem 1 that

$$
\int_{\Omega}\left|m_{h}-\Pi m_{h}\right|^{2} d x \leq c_{0}^{-1} \int_{\Omega} \phi\left(m_{h}\right) d x \leq c_{0}^{-1} E\left(m_{h}\right) \leq c_{0}^{-1} c_{1} h
$$

We next give an estimate for $m_{h 3}$ where $m_{h}=\left(m_{h 1}, m_{h 2}, m_{h 3}\right)$. 
Lemma 2. There exists a constant $c_{9}$, independent of $h$, such that

$$
\left|\int_{\Omega} m_{h 3}(x) \zeta(x) d x\right| \leq c_{9} h^{1 / 2}\|\zeta\|_{\mathcal{U}} \quad \text { for } \zeta \in \mathcal{U}
$$

Proof of Lemma 2. Let $\eta(x) \in C_{c}^{\infty}\left(\mathbb{R}^{3}\right)$ be such that $\eta(x)=1$ on $\Omega$ where $C_{c}^{\infty}\left(\mathbb{R}^{3}\right)$ is the set of $C^{\infty}\left(\mathbb{R}^{3}\right)$ functions with compact support. Since $C_{c}^{\infty}(\Omega)$ is dense in $\mathcal{U}$, we may assume that $\zeta \in C_{c}^{\infty}(\Omega)$. We may further assume that $\zeta$ has been extended by zero on $\mathbb{R}^{3}-\Omega$ so that $\zeta \in C^{\infty}\left(\mathbb{R}^{3}\right)$ with support in $\Omega$. Next, let $v(x)=\eta(x) \bar{v}(x)$ where

$$
\bar{v}\left(x_{1}, x_{2}, x_{3}\right)=\int_{0}^{x_{3}} \zeta\left(x_{1}, x_{2}, s\right) d s
$$

Then

$$
\frac{\partial v}{\partial x_{3}}=\zeta \quad \text { in } \Omega
$$

and

$$
\|\nabla v\|_{L^{2}\left(\mathbb{R}^{3}\right)} \leq c_{10}\|\zeta\|_{\mathcal{U}}
$$

By (2.3)

$$
\begin{aligned}
\left|\left(m_{h 3} \chi_{\Omega}, \zeta\right)\right| & =\left|\left(m_{h 3} \chi_{\Omega}, \frac{\partial v}{\partial x_{3}}\right)\right| \\
& \leq|(\nabla u, \nabla v)|+\left|\left(m_{h 1} \chi_{\Omega}, \frac{\partial v}{\partial x_{1}}\right)\right|+\left|\left(m_{h 2} \chi_{\Omega}, \frac{\partial v}{\partial x_{2}}\right)\right| \\
& \leq\left(\|\nabla u\|_{L^{2}\left(\mathbb{R}^{3}\right)}+\left\|m_{h 1}\right\|_{L^{2}(\Omega)}+\left\|m_{h 2}\right\|_{L^{2}(\Omega)}\right)\|\nabla v\|_{L^{2}\left(\mathbb{R}^{3}\right)} \\
& \leq\left(\sqrt{2} c_{1}^{1 / 2} h^{1 / 2}+c_{0}^{-1 / 2} c_{1}^{1 / 2} h^{1 / 2}+c_{0}^{-1 / 2} c_{1}^{1 / 2} h^{1 / 2}\right) c_{10}\|\zeta\|_{\mathcal{U}}
\end{aligned}
$$

We now can give the proof of Theorem 2 .

Proof of Theorem 2. We have that

$$
\begin{aligned}
& \left|\int_{\Omega}\left[F\left(x, m_{h}\right)-\frac{1}{2} F\left(x, e_{3}\right)-\frac{1}{2} F\left(x,-e_{3}\right)\right] d x\right| \\
& \leq\left|\int_{\Omega}\left[F\left(x, m_{h}\right)-F\left(x, \Pi m_{h}\right)\right] d x\right| \\
& \quad+\left|\int_{\Omega}\left[F\left(x, \Pi m_{h}\right)-\frac{1}{2} F\left(x, e_{3}\right)-\frac{1}{2} F\left(x,-e_{3}\right)\right] d x\right| \\
& =I_{1}+I_{2} .
\end{aligned}
$$


We will estimate $I_{1}$ and $I_{2}$ separately. We have from Lemma 1 that

$$
\begin{aligned}
I_{1} & \leq \int_{\Omega}\|F(x, .)\|_{L i p\left(\mathbb{S}^{2}\right)}\left|m_{h}-\Pi m_{h}\right| d x \\
& \leq\|F\|_{L^{2}\left(\Omega ; L i p\left(\mathbb{S}^{2}\right)\right)}\left\|m_{h}-\Pi m_{h}\right\|_{L^{2}(\Omega)} \\
& \leq c_{8}^{1 / 2}\|F\|_{L^{2}\left(\Omega ; L i p\left(\mathbb{S}^{2}\right)\right)} h^{1 / 2} .
\end{aligned}
$$

Denote $G(x)=F\left(x, e_{3}\right)-F\left(x,-e_{3}\right)$. Since

$$
\begin{aligned}
& F\left(x, \Pi m_{h}\right)-\frac{1}{2} F\left(x, e_{3}\right)-\frac{1}{2} F\left(x,-e_{3}\right) \\
& =\left\{\begin{aligned}
\frac{1}{2} G(x) & \text { if } \Pi m_{h}=e_{3} \\
-\frac{1}{2} G(x) & \text { if } \Pi m_{h}=-e_{3}
\end{aligned}\right.
\end{aligned}
$$

we have from Lemma 1 and Lemma 2 that

$$
\begin{aligned}
I_{2} & \leq\left|\int_{\Omega} \frac{1}{2} e_{3} \cdot \Pi m_{h} G d x\right| \\
& \leq \frac{1}{2}\left|\int_{\Omega} e_{3} \cdot\left(m_{h}-\Pi m_{h}\right) G d x\right|+\frac{1}{2}\left|\int_{\Omega} e_{3} \cdot m_{h} G d x\right| \\
& \leq \frac{1}{2}\left\|m_{h}-\Pi m_{h}\right\|_{L^{2}(\Omega)}\|G\|_{L^{2}(\Omega)}+\frac{1}{2}\left|\int_{\Omega} m_{3 h} G d x\right| \\
& \leq \frac{1}{2} c_{8}^{1 / 2} h^{1 / 2}\|G\|_{L^{2}(\Omega)}+\frac{1}{2} c_{9} h^{1 / 2}\|G\|_{\mathcal{U}} .
\end{aligned}
$$

The result follows from (4.21) and (4.22) .

Acknowledgements. The authors would like to thank Gene Fabes, David Kinderlehrer, and Dick James for useful discussions on the subject of this paper.

\section{REFERENCES}

[1] R. A. Adams, Sobolev Spaces, Academic Press, New York, 1975.

[2] Shmuel Agmon, Lectures on Elliptic Boundary Value Problems, Van Nostrand, Princeton, N. J., 1965.

[3] J. M. BALL, A version of the fundamental theorem for Young measures, in Partial Differential Equations and Continuum Models of Phase Transitions, Lecture Notes in Physics 344, M. Rascle, D.Serre, and M. Slemrod, eds., Springer-Verlag, Berlin, New York, 1989, pp. 207-215.

[4] J. M. Ball and R. D. James, Fine phase mixtures as minimizers of energy, Arch. Rational Mech. Anal., 100 (1987), pp. 13-52.

[5] J. M. Ball and R. D. James, Proposed experimental tests of a theory of fine microstructure and the two-well problem, preprint, 1990.

[6] W. F. Brown, Magnetostatic Principles in Ferromagnetism. Selected Topics in Solid State Physics, vol. 1, E.P. Wohlfarth, editor, North-Holland, 1962. 
[7] W. F. Brown, Micromagnetics, John Wiley and Sons, New York, 1963.

[8] W. F. BRown, Magnetoelastic Interactions, Tracts in Natural Philosophy, vol. 9, C. Truesdell, editor, Springer, New York, 1966.

[9] M. Снірот, Numerical analysis of oscillations in nonconvex problems, to appear.

[10] M. Chipot AND C. Collins, Numerical approximations in variational problems with potential wells, IMA Preprint 696.

[11] M. Chipot and D. Kinderlehrer, Equilibrium configurations of crystals, Arch. Rational Mech. Anal, 103 (1988), pp. 237-277.

[12] C. Collins, D. Kinderlehrer, and M. Luskin, Numerical approximation of the solution of a variational problem with a double well potential, SIAM J. Numer. Anal. (to appear).

[13] C. Collins and M. Luskin, The computation of the austenitic-martensitic phase transition, in Partial Differential Equations and Continuum Models of Phase Transitions, Lecture Notes in Physics 344, M. Rascle, D.Serre, and M. Slemrod, eds., Springer-Verlag, Berlin, New York, 1989, pp. 34-50.

[14] C. Collins AND M. Luskin, Computational results for phase transitions in shape memory materials, in Smart Materials, Structures, and Mathematical Issues, C. Rogers, ed., Technomic Publishing Co., Lancaster, Pennsylvania, 1989, pp. 198-215.

[15] C. Collins and M. Luskin, Numerical modeling of the microstructure of crystals with symmetry-related variants, in Proceedings of the ARO US-Japan Workshop on Smart/Intelligent Materials and Systems, Technomic Publishing Co., Lancaster, Pennsylvania, to appear.

[16] C. Collins And M. Luskin, Optimal order error estimates for the finite element approximation of the solution of a nonconvex variational problem, Minnesota Supercomputer Institute preprint 91-36.

[17] J. L. ERICKSEN, Some constrained elastic crystals, in Material Instabilities in Continuum Mechanics and Related Problems, J. M. Ball, ed., Oxford University Press, Oxford, 1987, pp. 119-137.

[18] J. L. Ericksen, Constitutive theory for some constrained elastic crystals, Int. J. Solids and Structures, 22 (1986), pp. 951-964.

[19] G. B. Folland, Introduction to partial differential equations, Princeton University Press, Princeton, N.J., 1976.

[20] I. Fonseca, Variational methods for elastic crystals, Arch. Rat. Mech. Anal., 97 (1985), pp. 189-220.

[21] I. FonSECA, The lower quasiconvex envelope of the stored energy function for an elastic crystal, J. Math. Pures et Appl., 67 (1988), pp. 175-195.

[22] D. A. FRENCH, On the convergence of finite element approximations of a relaxed variational problem, SIAM J. Numer. Anal. (1990), pp. 419-436.

[23] R. D. JAMEs, Microstructure and weak convergence, in Material Instabilities in Continuum Mechanics and Related Problems, J. M. Ball, ed., Oxford University Press, Oxford, 1987, pp. 175-196.

[24] R. D. JAMES, Relation between microscopic and macroscopic properties of crystals undergoing phase transformation, in Proc. 7th Army Conf. on Applied Mathematics and Computing, F. Dressel, editor, 1989.

[25] R. D. James and D. Kinderlehrer, An example of frustration in a ferromagnetic material, in Defects, Singularities, and Patterns in Nematic Liquid Crystals: Mathematical and Physical Aspects, Nato Meeting Series, J. M. Coron, F. Helein, J. M. Ghidaglia, eds., Kluwer Academic Publishers, Dordrecht, Netherlands, 1990.

[26] R. D. James and D. Kinderlehrer, Frustration in ferromagnetic materials, Continuum Mechanics and Thermodynamics, 2 (1990), pp. 215-239.

[27] D. Kinderlehrer, Remarks about equilibrium configurations of crystals, in Material Instabilities in Continuum Mechanics and Related Problems, J. M. Ball, ed., Oxford University Press, Oxford, 1987, pp. 217-242.

[28] R. KонN, The relationship between linear and nonlinear variational models of coherent phase transitions, in Proceedings of the Seventh Army Conference on Applied Mathematics and Computing, West Point, F. Dressel, ed., 1989.

[29] M. LUSKIN AND L. MA, The finite element computation of microstructure in micromagnetics, to appear.

[30] W. Rudin, Functional analysis, McGraw-Hill, New York, 1973.

[31] E. Stein, Singular Integrals and Differentiability Properties of Functions, Princeton University Press, Princeton, N.J., 1970. 
[32] L. TARTAR, Compensated compactness and applications to partial differential equations, in Nonlinear Analysis and Mechanics: Heriot-Watt Symposium IV, Research Notes 39, R. J. Knops, ed., Pitman, London, 1978, pp. 136-212. 


\section{Recent IMA Preprints}

Author/s

Title

Geneviève Raugel and George R. Sell, Navier-Stokes equations in thin 3d domains: Global regularity of solutions I

Emanuel Parzen, Time series, statistics, and information

Andrew Majda and Kevin Lamb, Simplified equations for low Mach number combustion with strong heat release

Ju. S. Il'yashenko, Global analysis of the phase portrait for the Kuramoto-Sivashinsky equation

James F. Reineck, Continuation to gradient flows

Mohamed Sami Elbialy, Simultaneous binary collisions in the collinear N-body problem

John A. Jacquez and Carl P. Simon, Aids: The epidemiological significance of two different mean rates of partner-change

Carl P. Simon and John A. Jacquez, Reproduction numbers and the stability of equilibria of SI models for heterogeneous populations

Matthew Stafford, Markov partitions for expanding maps of the circle

Ciprian Foias and Edriss S. Titi, Determining nodes, finite difference schemes and inertial manifolds

M.W. Smiley, Global attractors and approximate inertial manifolds for abstract dissipative equations

M.W. Smiley, On the existence of smooth breathers for nonlinear wave equations

Hitay Özbay and Janos Turi, Robust stabilization of systems governed by singular integro-differential equations

Mary Silber and Edgar Knobloch, Hopf bifurcation on a square lattice

Christophe Golé, Ghost circles for twist maps

Christophe Golé, Ghost tori for monotone maps

Christophe Golé, Monotone maps of $T^{n} \times R^{n}$ and their periodic orbits

E.G. Kalnins and W. Miller, Jr., Hypergeometric expansions of Heun polynomials

Victor A. Pliss and George R. Sell, Perturbations of attractors of differential equations

Avner Friedman and Peter Knabner, A transport model with micro- and macro-structure

E.G. Kalnins and W. Miller, Jr., A note on group contractions and radar ambiguity functions

George R. Sell, References on dynamical systems

Shui-Nee Chow, Kening Lu and George R. Sell, Smoothness of inertial manifolds

Shui-Nee Chow, Xiao-Biao Lin and Kening Lu, Smooth invariant foliations in infinite dimensional spaces

Kening Lu, A Hartman-Grobman theorem for scalar reaction-diffusion equations

Christophe Golé and Glen R. Hall, Poincaré's proof of Poincaré's last geometric theorem

Mario Taboada, Approximate inertial manifolds for parabolic evolutionary equations via Yosida approximations

Peter Rejto and Mario Taboada, Weighted resolvent estimates for Volterra operators on unbounded intervals

Joel D. Avrin, Some examples of temperature bounds and concentration decay for a model of solid fuel combustion

Susan Friedlander and Misha M. Vishik, Lax pair formulation for the Euler equation

H. Scott Dumas, Ergodization rates for linear flow on the torus

A. Eden, A.J. Milani and B. Nicolaenko, Finite dimensional exponential attractors for semilinear wave equations with damping

A. Eden, C. Foias, B. Nicolaenko \& R. Temam, Inertial sets for dissipative evolution equations

A. Eden, C. Foias, B. Nicolaenko \& R. Temam, Hölder continuity for the inverse of Mañés projection

Michel Chipot and Charles Collins, Numerical approximations in variational problems with potential wells

Huanan Yang, Nonlinear wave analysis and convergence of MUSCL schemes

László Gerencsér and Zsuzsanna Vágó, A strong approximation theorem for estimator processes in continuous time

László Gerencsér, Multiple integrals with respect to $L$-mixing processes

David Kinderlehrer and Pablo Pedregal, Weak convergence of integrands and the Young measure representation

701 Bo Deng, Symbolic dynamics for chaotic systems

P. Galdi, D.D. Joseph, L. Preziosi, S. Rionero, Mathematical problems for miscible, incompressible fluids with Korteweg stresses

Charles Collins and Mitchell Luskin, Optimal order error estimates for the finite element approximation of the solution of a nonconvex variational problem

Peter Gritzmann and Victor Klee, Computational complexity of inner and outer j-radii of polytopes in finite-dimensional normed spaces

A. Ronald Gallant and George Tauchen, A nonparametric approach to nonlinear time series analysis: estimation and simulation 
H.S. Dumas, J.A. Ellison and A.W. Sáenz, Axial channeling in perfect crystals, the continuum model and the method of averaging

M.A. Kaashoek and S.M. Verduyn Lunel, Characteristic matrices and spectral properties of evolutionary systems

Xinfu Chen, Generation and Propagation of interfaces in reaction diffusion systems

Avner Friedman and Bei Hu, Homogenization approach to light scattering from polymer-dispersed liquid crystal films

Yoshihisa Morita and Shuichi Jimbo, ODEs on inertial manifolds for reaction-diffusion systems in a singularly perturbed domain with several thin channels

Wenxiong Liu, Blow-up behavior for semilinear heat equations:multi-dimensional case

Hi Jun Choe, Hölder continuity for solutions of certain degenerate parabolic systems

Hi Jun Choe, Regularity for certain degenerate elliptic double obstacle problems

Fernando Reitich, On the slow motion of the interface of layered solutions to the scalar Ginzburg-Landau equation

Xinfu Chen and Fernando Reitich, Local existence and uniqueness of solutions of the Stefan problem with surface tension and kinetic undercooling rings

W. Balser, B.L.J. Braaksma, J.-P. Ramis and Y. Sibuya, Multisummability of formal power series solutions of linear ordinary differential equations

Peter J. Olver and Chehrzad Shakiban, Dissipative decomposition of partial differential equations

Clark Robinson, Homoclinic bifurcation to a transitive attractor of Lorenz type, II

Michelle Schatzman, A simple proof of convergence of the $Q R$ algorithm for normal matrices without shifts

Ian M. Anderson, Niky Kamran and Peter J. Olver, Internal, external and generalized symmetries

C. Foias and J.C. Saut, Asymptotic integration of Navier-Stokes equations with potential forces. I

Ling Ma, The convergence of semidiscrete methods for a system of reaction-diffusion equations

Adelina Georgescu, Models of asymptotic approximation

A. Makagon and H.Salehi, On bounded and harmonizable solutions on infinite order arma systems

San-Yih Lin and Yan-Shin Chin, An upwind finite-volume scheme with a triangular mesh for conservation laws

J.M. Ball, P.J. Holmes, R.D. James, R.L. Pego \& P.J. Swart, On the dynamics of fine structure

KangPing Chen and Daniel D. Joseph, Lubrication theory and long waves

J.L. Ericksen, Local bifurcation theory for thermoelastic Bravais lattices

Mario Taboada and Yuncheng You, Some stability results for perturbed semilinear parabolic equations

A.J. Lawrance, Local and deletion influence

Bogdan Vernescu, Convergence results for the homogenization of flow in fractured porous media

Xinfu Chen and Avner Friedman, Mathematical modeling of semiconductor lasers

Yongzhi Xu, Scattering of acoustic wave by obstacle in stratified medium

Songmu Zheng, Global existence for a thermodynamically consistent model of phase field type

Heinrich Freistühler and E. Bruce Pitman, A numerical study of a rotationally degenerate hyperbolic system part I: the Riemann problem

Epifanio G. Virga, New variational problems in the statics of liquid crystals

Yoshikazu Giga and Shun'ichi Goto, Geometric evolution of phase-boundaries

Ling Ma, Large time study of finite element methods for 2D Navier-Stokes equations

Mitchell Luskin and Ling Ma, Analysis of the finite element approximation of microstructure in micromagnetics

M. Chipot, Numerical analysis of oscillations in nonconvex problems

J. Carrillo and M. Chipot, The dam problem with leaky boundary conditions

Eduard Harabetian and Robert Pego, Efficient hybrid shock capturing schemes

B.L.J. Braaksma, Multisummability and Stokes multipliers of linear meromorphic differential equations

Tae Il Jeon and Tze-Chien Sun, A central limit theorem for non-linear vector functionals of vector Gaussian processes

Chris Grant, Solutions to evolution equations with near-equilibrium initial values

Mario Taboada and Yuncheng You, Invariant manifolds for retarded semilinear wave equations

Peter Rejto and Mario Taboada, Unique solvability of nonlinear Volterra equations in weighted spaces

Hi Jun Choe, Holder regularity for the gradient of solutions of certain singular parabolic equations

Jack D. Dockery, Existence of standing pulse solutions for an excitable activator-inhibitory system

Jack D. Dockery and Roger Lui, Existence of travelling wave solutions for a bistable evolutionary ecology model

Giovanni Alberti, Luigi Ambrosio and Giuseppe Buttazzo, Singular perturbation problems with a compact support semilinear term 